\title{
Multistable behavior above synchronization in a locally coupled Kuramoto model
}

\author{
Paulo F. C. Tilles, ${ }^{1}$ Fernando F. Ferreira, ${ }^{2}$ and Hilda A. Cerdeira ${ }^{1}$ \\ ${ }^{1}$ Instituto de Física Teórica UNESP - Universidade Estadual Paulista, Rua Dr. Bento Teobaldo Ferraz, 271, Bloco II, \\ Barra Funda, 01140-070 São Paulo, Brazil \\ ${ }^{2}$ Grupo Interdisciplinar de Física da Informação e Economia GRIFE, Escola de Arte, Ciências e Humanidades, Universidade de São Paulo, \\ Avenida Arlindo Bettio 1000, 03828-000 São Paulo, Brazil \\ (Received 1 November 2010; revised manuscript received 10 February 2011; published 14 June 2011)
}

\begin{abstract}
A system of nearest neighbors Kuramoto-like coupled oscillators placed in a ring is studied above the critical synchronization transition. We find a richness of solutions when the coupling increases, which exists only within a solvability region (SR). We also find that the solutions possess different characteristics, depending on the section of the boundary of the SR where they appear. We study the birth of these solutions and how they evolve when the coupling strength increases, and determine the diagram of solutions in phase space.
\end{abstract}

DOI: 10.1103/PhysRevE.83.066206

PACS number(s): 05.45.Xt, 05.45.Jn

\section{INTRODUCTION}

The ubiquity of phenomena linked to coupled systems which can behave chaotically has made them the focus of interest for the last 20 years. The study of these systems has raised interest with the intent of realistically modeling spatially extended systems, as diverse as Josephson junction arrays, multimode lasers, vortex dynamics, biological information processing, and neurodynamics as well as applications in communications [1-8], with the belief that dominant features will be retained in such simple models. Coupled systems with local interactions are of special importance. In particular the Kuramoto model [9] in its local version, i.e., the locally coupled Kuramoto model (LCKM), where individually linear oscillators behave chaotically under the effect of nonlinear local interactions, has raised attention since most features of systems with phase coupling appear in this particularly simple model [10-14].

This system which shows synchronization in the mean frequency has been thoroughly studied at and before full synchronization. The oscillators cluster in average frequency, decreasing the number of clusters until they fuse into a single one at full synchronization. At this moment the frequency becomes constant and the phases lock, such that all phase differences are constant. The solution for full synchronization and its stability has been studied by many authors [15-19]. Zheng et al. [12] already in 1998 pointed out that the behavior of the order parameter "indicates the coexistence of multiple attractors of phase locking states" above the synchronization critical coupling, $K_{s}$. Even when synchronization with coexistence of attractors has been reported by different authors and in different fields below $K_{s}$ [12-14,20-25], nobody, to the best of our knowledge, has pursued the matter above full synchronization. If we have the intention to simulate real systems, mostly in technological applications, it is important to know whether we can move freely below and also above synchronization within stable solutions and to know whether they are unique. In this work we shall study a LCKM above complete synchronization and show that there is an unexpected richness of behavior: multistable solutions appear and we cannot change the strength of the coupling without danger of falling into different attractors.

In Sec. II we present the model and discuss the conditions for the existence of multistable solutions; in Sec. III we address their stability and estimate their basins of attraction; in Sec. IV we show how they appear, starting from a chain of oscillators; finally in Sec. V we summarize the work.

\section{LOCALLY COUPLED KURAMOTO MODEL IN THE SYNCHRONIZED REGION}

The model consists of a set of linear oscillators placed in a ring, with a nonlinear nearest-neighbor interaction described by the following equations:

$$
\dot{\theta}_{n}=\omega_{n}+K\left[\sin \left(\theta_{n-1}-\theta_{n}\right)+\sin \left(\theta_{n+1}-\theta_{n}\right)\right],
$$

where $n=1, \ldots, N$, and $\omega_{n} \in\{\omega\}$ is the set of natural frequencies of the oscillators. The ring topology is defined by the periodic conditions $\theta_{N+1}=\theta_{1}$ and $\theta_{0}=\theta_{N}$. There is a minimum value for the coupling constant $K$, denoted as critical synchronization coupling $K_{s}$, that drives the system into a fully synchronized state [16-18]. In this state the oscillators' instantaneous frequencies assume a constant value $\Omega=\frac{1}{N} \sum_{j=1}^{N} \omega_{j}$ that remains unchanged for any $K \in\left[K_{s}, \infty\right)$.

The set of equations (1) in a synchronized state may be written as

$$
\frac{\Omega-\omega_{n}}{K}=\sin \phi_{n-1}-\sin \phi_{n}, \quad n=1, \ldots, N,
$$

where $\phi_{n}=\theta_{n}-\theta_{n+1}$. The solutions depend on the number of oscillators $N$ and the coupling constant $K \geqslant K_{s}$. We shall see that $K_{s}$ will depend on a particular variable $\phi_{n^{*}}$, where $n^{*}$ plays a very important role which will be apparent later. For a given distribution of frequencies $\{\omega\}$, any $\phi_{n}$ may be written as a function of an arbitrarily chosen variable $\phi_{n^{*}}$ on the synchronization region:

$$
\phi_{n}\left(\phi_{n^{*}}, K\right)=\arcsin \left[\sin \phi_{n^{*}}+\frac{1}{K} \sum_{j=n+1}^{n^{*}}\left(\Omega-\omega_{j}\right)\right] \text {, }
$$

for $n=1, \ldots, n^{*}-1$ and

$$
\phi_{n}\left(\phi_{n^{*}}, K\right)=\arcsin \left[\sin \phi_{n^{*}}-\frac{1}{K} \sum_{j=n^{*}+1}^{n}\left(\Omega-\omega_{j}\right)\right] \text {, }
$$

for $n=n^{*}+1, \ldots, N-1$. Since the sum of all phase differences is equal to zero, it is possible to write $\phi_{N}=-\sum_{j=1}^{N-1} \phi_{j}$, 
in order to reduce the set of $N$ equations (2) to a single one depending on two variables $\left(\phi_{n^{*}}, K\right)$ :

$$
\sin \left(\phi_{n^{*}}+\sum_{n \neq n^{*}}^{N-1} \phi_{n}\right)+\sin \phi_{n^{*}}=\frac{\sum_{j=1}^{n^{*}}\left(\omega_{j}-\Omega\right)}{K} .
$$

The choice of $\phi_{n^{*}}$ will become clear in the next paragraph.

If one removes a single link (interaction) between any pair of oscillators the result is a chain with free ends. After repeating the procedure for each possible link we obtain $N$ distinct chains. If we can find the chain encapsulated inside the ring with the highest critical synchronization coupling we may be able to find the solutions above $K_{s}$. We approach this task by applying Strogatz and Mirollo's calculation [15] successively, so that at the synchronization threshold the coupling strength for each chain is given by

$$
K_{r}=\max _{r \leqslant l<r+N}\left|\sum_{j=r}^{l}\left(\Omega-\omega_{j}\right)\right|, \quad r=1, \ldots, N .
$$

Here $r$ denotes the shift on the indices of the oscillators from where the chain starts, and $\omega_{j}$, for $j>N$, is defined by the periodic boundary conditions on the natural frequencies, $\omega_{r+N}=\omega_{r}$ for $r=1, \ldots, N$. For every value of $r$ we have to determine the maximum $K_{r}$, which defines $l_{r}$. From $K_{r}$ we obtain a sequence $\left\{K_{1}, \ldots, K_{N}\right\}$. The maximum value in this sequence defines $K_{s}^{\text {chain }}=K_{r_{\max }}$ with index $l_{r_{\max }}$. As it is always possible to relabel the oscillators in the ring while keeping their specific ordering, we shall consider the label obtained from the translation $n \rightarrow n+1-r_{\max }$. On this labeling the synchronization coupling is written as $K_{s}^{\text {chain }}=$ $\left|\sum_{j=1}^{n^{*}}\left(\Omega-\omega_{j}\right)\right|$, identifying $n^{*}=l_{r_{\max }}+1-r_{\max }$.

Once we established our basic assumptions, we write Eq. (4) as

$$
\sin \phi_{n^{*}}-\sin \left[\phi_{N}\left(n^{*}, K\right)\right]=s \frac{K_{s}^{\text {chain }}}{K},
$$

with $s=\operatorname{sgn}\left[\sum_{j=1}^{n^{*}}\left(\Omega-\omega_{j}\right)\right]$, and solve it numerically using Mathematica. The general form of the solution is presented in Fig. 1 for $N=10,20$, and 50, where the frequencies $\{\omega\}_{N}$ were generated from a uniform distribution function defined in the interval $[-10,10]$. We have performed calculations for different natural frequency realizations, all the results present the same features as will be described, therefore we keep here to a single realization for a given $N$ to avoid confusion.

We see that there are multiple phase locking solutions for the system above $K_{s}$, spontaneously generated within a confined region on the bifurcation diagram (BD), as indicated by the dotted lines. These solutions are of two kinds: (a) type I solutions generated on the bottom boundary of BD with $\sin \phi_{n^{*}} \neq s$ and bifurcate into branches keeping $\operatorname{sgn}\left(\cos \phi_{n^{*}}\right)$ invariant; (b) type II solutions born near the top boundary of $\mathrm{BD}$ (close to $\sin \phi_{n^{*}}=s$ ) and the branches have opposite signs of $\cos \phi_{n^{*}}$.
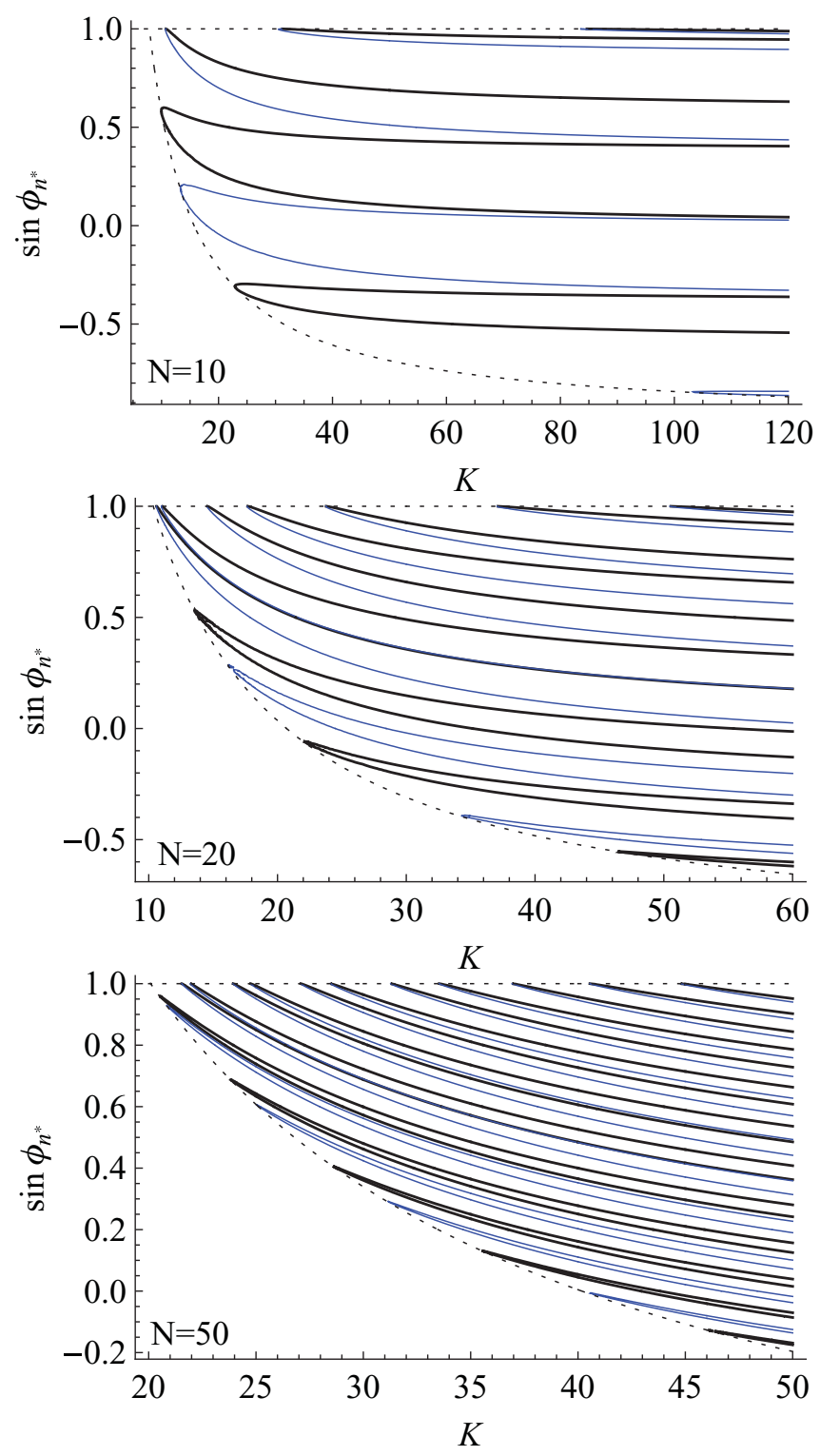

FIG. 1. (Color online) Numerical solutions of Eq. (4) representing the bifurcation diagram for a ring of oscillators (stability not explicit): Top: $N=10$ with natural frequencies $\omega_{1}=6.9, \omega_{2}=2.8, \omega_{3}=$ $-0.4, \omega_{4}=-2.6, \omega_{5}=1.3, \omega_{6}=-6.8, \omega_{7}=0.8, \omega_{8}=-1.6, \omega_{9}=$ -9.5 , and $\omega_{10}=-6.7$. Middle: realization with $N=20$. Bottom: realization with $N=50$. The sets of natural frequencies $\{\omega\}_{N}$ are generated from a uniform distribution function defined in the interval $[-10,10]$. Thick (black) lines represent solutions with $\cos \phi_{n^{*}}>0$, thin (blue) lines have $\cos \phi_{n^{*}}<0$, and the dotted lines are the limiting boundaries $\sin \phi_{N}=-s$ and $\sin \phi_{n^{*}}=s$.

The confinement region may be obtained, since for each value of $\sin \phi_{n^{*}}$ there is a maximum value of $K$ below which $\mathrm{Eq},(6)$ is never satisfied. We assume that $\sin \phi_{N}=-s$ and find that all solutions appear at

$$
\left|\sin \phi_{n^{*}}\right| \geqslant\left|s\left(\frac{K_{s}^{\text {chain }}}{K}-1\right)\right| \text {. }
$$

The equal sign describes the points where type I solutions touch this bordering line and is represented by the inferior dotted line in the BD. The solvability region (SR) contains all 
the synchronized solutions and it can be defined by Eq. (7). It is worth mentioning that Eq. (7) shows that the critical synchronization coupling for the ring satisfies the condition $K_{s} \geqslant \frac{K_{s}^{\text {chain }}}{2}$.

A closer look at the BD shows that there is always one solution from each bifurcation that is tangent to the boundary of the SR at only one point (this will appear clearly when we discuss Fig. 5): it corresponds to $\sin \phi_{N}=-s$ for type I and $\sin \phi_{n^{*}}=s$ for type II solutions. On all of these tangent points, independent of the type of boundary, a straightforward calculation shows that they satisfy the condition

$$
\cos \left(\sum_{n \neq n^{*}}^{N-1} \phi_{n}\right)=\frac{K_{s}^{\text {chain }}}{K}-1 .
$$

The number of bifurcations depends on the number of solutions of Eq. (8) over each boundary of the SR. Since the cosine argument may be expanded as a Laurent series defined by $\phi_{n}=A_{0}+\sum_{m=1}^{\infty} \frac{A_{m}^{(n)}}{K^{m}}$, the decaying behavior as a function of $K$ guarantees a finite number of solutions. From the definition of the phase differences (3), it is possible to see that the $A_{0}$ term depends on the size of the system so that the effect of increasing the number of oscillators also increases the number of phase locking solutions above $K_{s}$, as may be observed in Fig. 1 .

One way of addressing the question of how the multiple solutions are generated is to look at the solutions of $\phi_{n^{*}}$ and $\phi_{N}$ on the tangent points. By starting with type I solutions the condition $\sin \phi_{N}=-s$ imposes that $\phi_{N}$ should satisfy the equation

$$
\phi_{N}^{(\mathrm{I})}\left(m_{1}\right)=-s \frac{\left(4 m_{1}+1\right) \pi}{2}, m_{1}=0,1,2, \ldots
$$

For each possible value of $m_{1}$ Eq. (6) admits two solutions:

$$
\begin{gathered}
\phi_{n^{*}}^{(\mathrm{I}, 1)}=\arcsin \left[s\left(\frac{K_{s}^{\text {chain }}}{K}-1\right)\right], \\
\phi_{n^{*}}^{(\mathrm{I}, 2)}=-\arcsin \left[s\left(\frac{K_{s}^{\text {chain }}}{K}-1\right)\right]+\pi .
\end{gathered}
$$

If we fix $\phi_{n^{*}}=\phi_{n^{*}}^{(\mathrm{I}, 1)}$ then the values assumed by $m_{1}$ provide all the values of $K$ where the solutions with $\cos \phi_{n^{*}}>0$ are tangent to the limiting boundary. Similarly when $\phi_{n^{*}}=\phi_{n^{*}}^{(\mathrm{I}, 2)}$ the values of $m_{1}$ give the tangent points for the solutions with $\cos \phi_{n^{*}}<0$. Figure 2 (top) shows these solutions for the $N=$ 10 configuration used previously.

The same procedure may be performed for the type II solutions, where the condition $\sin \phi_{n^{*}}=s$ imposes

$$
\phi_{n^{*}}^{(\mathrm{II})}\left(m_{2}\right)=-s \frac{\left(4 m_{2}-1\right) \pi}{2}, m_{2}=0,1,2, \ldots
$$

Now for each value of $m_{2}$ we also have two types of solutions for $\phi_{N}$ :

$$
\begin{gathered}
\phi_{N}^{(\mathrm{II}, 1)}=-\arcsin \left[s\left(\frac{K_{s}^{\text {chain }}}{K}-1\right)\right], \\
\phi_{N}^{(\mathrm{II}, 2)}=\arcsin \left[s\left(\frac{K_{s}^{\text {chain }}}{K}-1\right)\right]-\pi .
\end{gathered}
$$
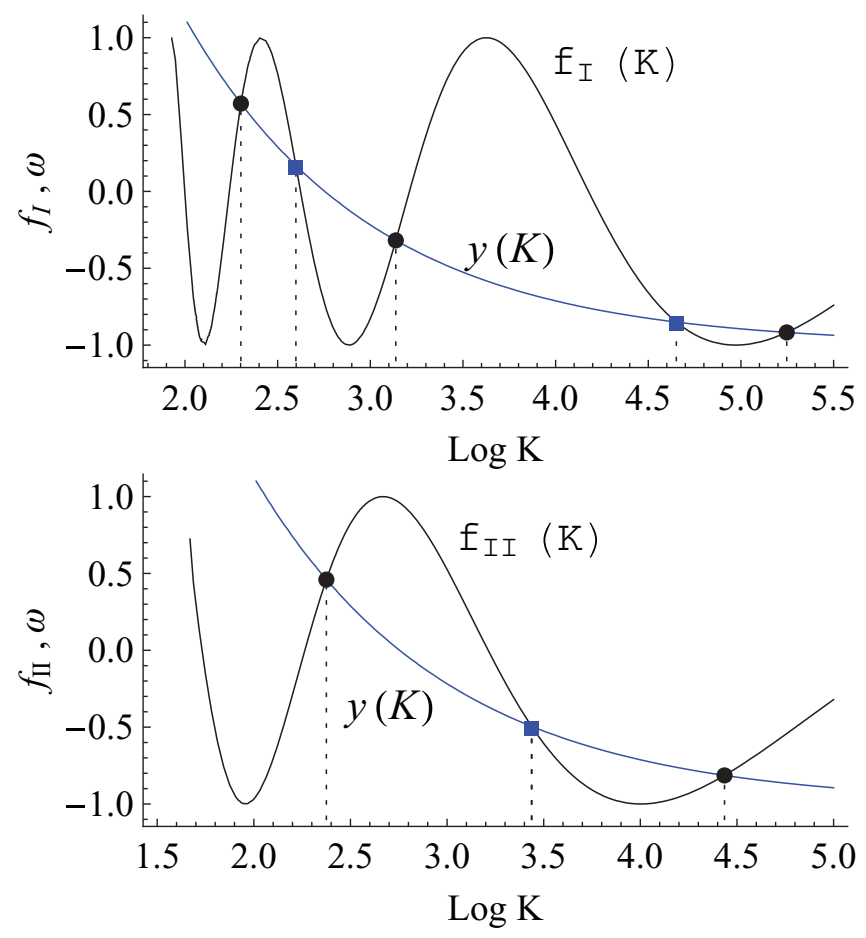

FIG. 2. (Color online) Graphical representation of the numerical solutions of Eq. (8) for the $N=10$ realization, with $y=s\left(\frac{K_{s}^{\text {chain }}}{K}-1\right)$ (thin blue curve) and $f_{\mathrm{I}, \mathrm{II}}=\cos \left(\sum_{n \neq n^{*}}^{N-1} \phi_{n}\right)$ (thick black curve) calculated on the tangent points from type I and II solutions, respectively. Top: $\phi_{n^{*}}^{(\mathrm{I}, 1)}$ solutions (black dots) at $K_{\left(m_{1}=0\right)}=9.9905$, $K_{\left(m_{1}=1\right)}=23.0467, K_{\left(m_{1}=2\right)}=190.016$, and $\phi_{n^{*}}^{(\mathrm{I}, 2)}$ solutions (blue squares) at $K_{\left(m_{1}=0\right)}=13.443$ and $K_{\left(m_{1}=1\right)}=104.7171$. Bottom: $\phi_{N}^{\text {(II, } 1)}$ solutions (black dots) at $K_{\left(m_{2}=0\right)}=10.75473, K_{\left(m_{2}=1\right)}=84.3981$, and $\phi_{N}^{(\text {II, 2) }}$ solutions (blue squares) at $K_{\left(m_{2}=2\right)}=31.1029$.

The values of $K$ for the tangent points on this boundary are also shown in Fig. 2 (bottom), for the same realization with $N=10$.

With the description of all multiple phase locking solutions above $K_{s}$ (at least on the tangent points) it is possible to have some insight of their origin. We notice that the sum of the phase differences in Eq. (4) goes beyond $2 \pi$ but not as a multiple of $2 \pi$. In this way it is the presence of this term-connecting the first to the last oscillators of the chain-that generates the multiple solutions. The notable symmetry between the type I and type II tangent point solutions comes from the fact that if we remove the interaction term between oscillators $\theta_{1}$ and $\theta_{N}$ the critical synchronization coupling of the resulting chain is the same as the one obtained from extracting the interaction term connecting oscillators $\theta_{n^{*}}$ and $\theta_{n^{*}+1}$, even when the phase locked solutions may be different (this feature may be observed by reproducing the calculation described to obtain $K_{s}^{\text {chain }}$ starting with the label $n^{*}$ ). In spite of this symmetry the number of type I and type II solutions are in general not the same.

\section{STABILITY OF SOLUTIONS AND BASINS OF ATTRACTION}

To perform a linear stability analysis of the solutions it is necessary to obtain the Jacobian matrix, but as the equations of 
motion are invariant by global phase translations $\theta_{n} \rightarrow \theta_{n}+\Theta$ (for every $n$ ) the analysis is a little more complicated.

It is necessary to realize that the freedom of gauge reduces the $N$ equations of motion (1) to an $(N-1)$-dimensional system (this is an effect of the constraint $\sum_{n=1}^{N} \phi_{n}=0$ ). Although a gauge fixing condition would enable us to eliminate the extra degree of freedom and perform the stability analysis on the $\theta_{n}$ variables we believe there is a more tractable way to do this. Instead of considering the equations of motion as presented in Eq. (1) we write the equations on the $\phi_{n}$ variables and eliminate one degree of freedom, replacing $\phi_{N}$ by $-\sum_{n=1}^{N-1} \phi_{n}$. Because only the equations for $\dot{\phi}_{1}$ and $\dot{\phi}_{N-1}$ are dependent on $\phi_{N}$, we have

$$
\begin{gathered}
\dot{\phi}_{1}=\omega_{1}-\omega_{2}-K\left[\sin \left(\sum_{n=1}^{N-1} \phi_{n}\right)+2 \sin \phi_{1}-\sin \phi_{2}\right], \\
\dot{\phi}_{N-1}=\omega_{N-1}-\omega_{N}+K\left[\sin \left(\sum_{n=1}^{N-1} \phi_{n}\right)+\sin \phi_{N-2}\right. \\
\left.-2 \sin \phi_{N-1}\right] .
\end{gathered}
$$

The rest of the equations for $n=2, \ldots, N-2$ do not depend on $\phi_{N}$ and are expressed in the usual form

$$
\begin{aligned}
\dot{\phi}_{n}= & \omega_{n}-\omega_{n+1} \\
& +K\left(\sin \phi_{n-1}-2 \sin \phi_{n}+\sin \phi_{n+1}\right) .
\end{aligned}
$$

Due to the structure of the equations, the elements of the Jacobian matrix may be written as $J_{n, m}=\partial_{\phi_{m}} \dot{\phi}_{n}$. Since most of the elements are null, let us focus on the nonzero ones: the first and last lines are complete with the elements given by

$$
\begin{aligned}
J_{1,1}=-K\left[2 \cos \phi_{1}+\cos \left(\sum_{j=1}^{N-1} \phi_{j}\right)\right], \\
J_{1,2}=K\left[\cos \phi_{1}-\cos \left(\sum_{j=1}^{N-1} \phi_{j}\right)\right], \\
J_{N-1, N-2}=K\left[\cos \phi_{N-2}-\cos \left(\sum_{j=1}^{N-1} \phi_{j}\right)\right], \\
J_{N-1, N-1}=-K\left[2 \cos \phi_{N-1}+\cos \left(\sum_{j=1}^{N-1} \phi_{j}\right)\right],
\end{aligned}
$$

and for $n=1, \ldots, N-3$

$$
J_{N-1, n}=J_{1, n+2}=-k \cos \left(\sum_{j=1}^{N-1} \phi_{j}\right)
$$

all other lines from $n=2, \ldots, N-2$ have nonzero elements only on the diagonal and first neighbors, namely,

$$
J_{n, m}=K \cos \phi_{m}\left(\delta_{m, n-1}-2 \delta_{m, n}+\delta_{m, n+1}\right) .
$$

The linear stability of the fixed points is determined by the structure of the Jacobian eigenvalues: a solution will be stable

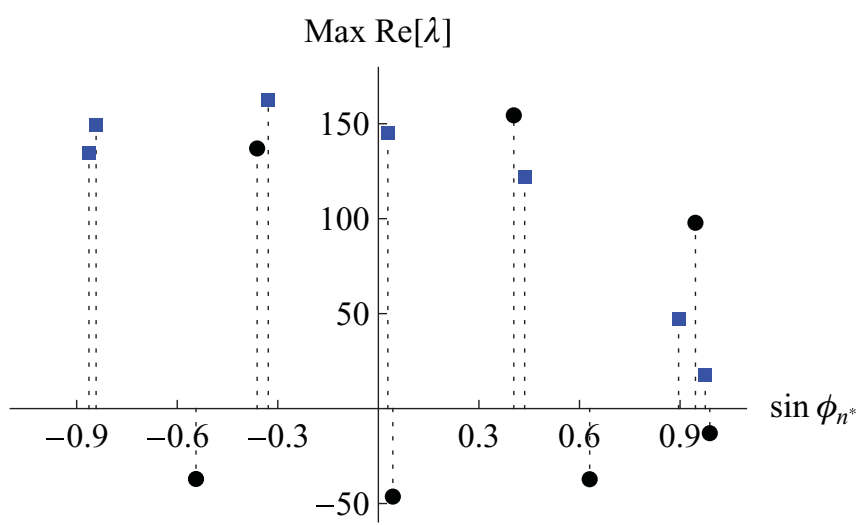

FIG. 3. (Color online) Largest Jacobian eigenvalue for the $N=$ 10 case with $K=120$. Black dots represent phase locking solutions with $\cos \phi_{n^{*}}>0$ while blue squares represent phase locking solutions with $\cos \phi_{n^{*}}<0$.

only if the real part of all eigenvalues are negative. Since a general system with $N$ oscillators has an $(N-1) \times(N-1)$ Jacobian matrix, the eigenvalue equation is too complicated to be treated analytically. In this way we perform a numerical approach that consists on fixing a value for the coupling $K$ and replacing the phase locked solutions $\phi_{n}$ on the Jacobian matrix elements by the numerical calculation of the eigenvalues $\lambda_{n}$, for $n=1, \ldots, N-1$. Figure 3 shows the real part of the largest eigenvalue for all fixed points for the case $N=10$ with $K=$ 120 (the fixed points may be inferred from either Fig. 1 or Fig. 4). By repeating the procedure for other values of $K$ on
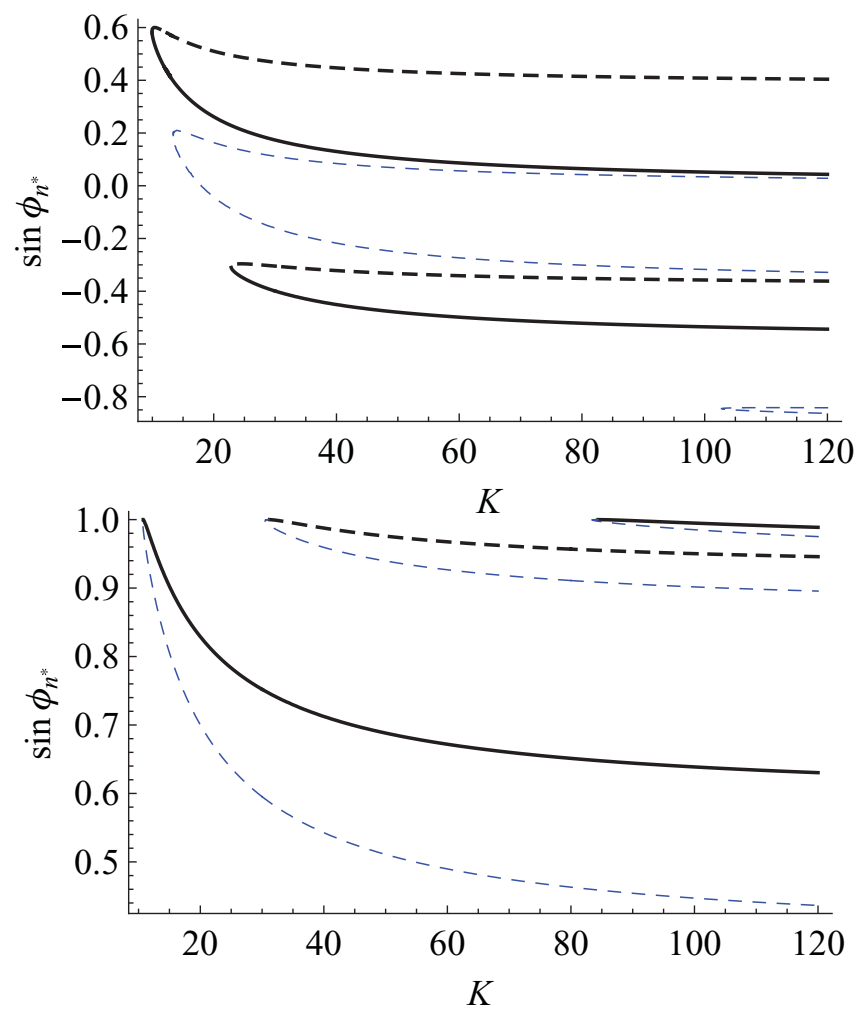

FIG. 4. (Color online) Bifurcation diagram for $N=10$ with explicit stability. Continuous (dashed) curves represent stable (unstable) solutions. Top: type I solutions. Bottom: type II solutions. 

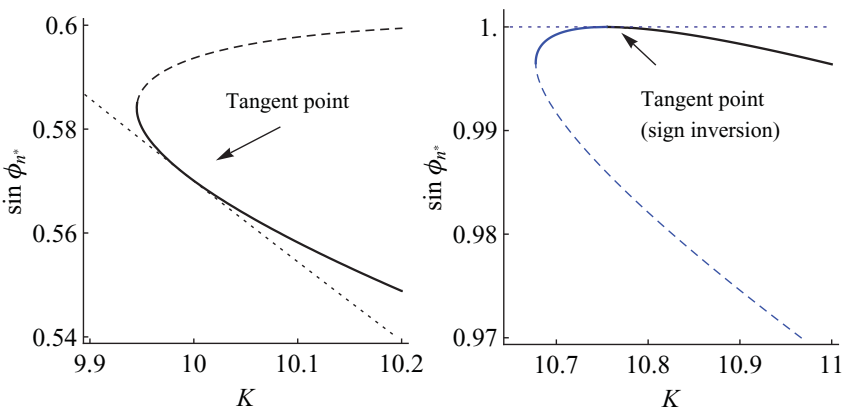

FIG. 5. (Color online) Zoom of the first two bifurcations present on the BD of the $N=10$ case. Left: Zoom of the $K_{s}$ bifurcation from Fig. 4 (top) with a tangent point from the stable solution touching the SR boundary at $\sin \phi_{N}=-s$. Right: first bifurcation from type II solutions from Fig. 4 (bottom) showing that the stable solution is actually born with $\cos \phi_{n^{*}}<0$ and changes sign when it touches the SR curve $\sin \phi_{n^{*}}=s$.

the range $\left[K_{s}, 120\right]$ we were able to determine the stability of all solutions, as shown on the BD from Fig. 4. Our results confirm that the stability of a branch is not altered by variations of $K$, therefore the results obtained from Fig. 3 may be extrapolated to the whole BD.

Let us focus on the $N=10$ case. If we look at the type I solutions (boundary of the SR with $\sin \phi_{n^{*}} \neq s$ ), as shown at the top of Fig. 4, we observe that if $\cos \phi_{n^{*}}>0$, two branches are born on a saddle node bifurcation and the stable branch is tangent to the boundary curve, $\sin \phi_{N}=-s$ (details are shown in Fig. 5 left). On the other hand both branches with $\cos \phi_{n^{*}}<$ 0 born at the same point, are unstable with at least one positive eigenvalue. If we take a look at the bottom of Fig. 4 it is possible to see that the type II solutions (close to $\sin \phi_{n^{*}}=s$ ) also present bifurcations that generate two unstable solutions. The stable solutions also have a point tangent to a SR curve $\left(\sin \phi_{n^{*}}=s\right)$, but since $\sin \phi_{n^{*}}=s, \cos \phi_{n^{*}}$ changes sign at the tangent point, which is different from the type I case, and the stable solution starts at the bifurcation with $\cos \phi_{n^{*}}<0$ and changes sign at the $\sin \phi_{n^{*}}=s$ line.

Now let us analyze the solutions near the bifurcations. All branches are born at local minima of the function $K\left(\phi_{n^{*}}\right)$ defined in the synchronized region $\left(\dot{\theta}_{n}=\Omega\right)$, where the critical synchronization coupling $K_{s}$ is the absolute minimum. Bearing this in mind, when we take a closer look at bifurcations from each type of solution, an interesting feature is observed (Fig. 5): $\sin \phi_{n}$ is not equal to \pm 1 at $K_{s}$ or at any other local minima for any $n$. The explanation for this behavior lies in Eq. (6): in order to have a bifurcation with a sine equal to \pm 1 it is necessary that either $\phi_{n^{*}}$ or $\phi_{N}$ present this property, but since $\phi_{N}$ is a nonlinear function of $\phi_{n^{*}}$ there are accessible synchronized solutions prior to the appearance of the sine equal to \pm 1 . This result is in agreement with the condition

$$
\sum_{n=1}^{N} \frac{1}{\cos \phi_{n}}=0,
$$

found to be satisfied at the critical coupling $K_{s}$ by any random distributed natural frequency on a ring (as shown in [17]), which makes it impossible to have a bifurcation with a sine equal to \pm 1 [27]. Nevertheless there is a sine equal to \pm 1 near the bifurcation, with the difference becoming smaller as $N \rightarrow \infty$ in agreement with previous literature where this fact has played a crucial role $[15,16]$. In the next section we will show how these deviations and the multiple solutions are generated from a chain of oscillators.

The general picture we obtained for the $\mathrm{BD}$ with random distributed frequencies, from both simulation and numerical calculation, may be summarized as follows: for a given $N$, a configuration $\{\omega\}_{N}$ determines a SR for the system in which all solutions come from bifurcations near the solvability boundaries; all stable solutions correspond to branches that touch the solvability boundary; it is possible to extrapolate the definition of the two types of solutions to the bifurcations themselves and label them as $K_{j}^{l}$, where $l=\mathrm{I}$ or II, for the type I or type II bifurcations and $j=1,2, \ldots$, gives the order of the minima; all bifurcations are saddle-node type, but odd values of $j$ lead to branches with stable nodes while even numbered ones generate only unstable solutions [26]; none of the bifurcations may appear with a sine equal to 1 because condition (15) must always be satisfied; in addition to the stability analysis our numerical simulations showed that every stable tangent point satisfies the condition $\partial_{K} \cos \left(\sum_{n \neq n^{*}}^{N-1} \phi_{n}\right)>0$, although we are not able to explain why it happens at this moment.

With the stability of the solutions fully described we turn our attention to the basin of attraction of the stable solutions. Since a general LCKM is a high dimensional system a graphical analysis becomes extremely difficult. To outline these difficulties we consider a statistical approach: given that the fixed points are represented by values of $\sin \phi_{n^{*}}$ we generate a large sample of random initial conditions for the phases on the interval $[-\pi, \pi]$, for fixed values of $K$, and estimate the size of the basin of attraction by the probability of the system to reach each of the stable solutions.

If we start with the case $N=10$ we could observe that as long as the values assumed by $K$ lie in a region where the number of stable solutions is kept constant, the size of the basins of attraction presents only small statistical fluctuations, which leads us to believe that these variations of $K$ have little effect on the size of a basin. When we vary the coupling constant across regions with increasing number of solutions we observe that when a stable fixed point is created its basin of attraction steals the majority of its size from the closest fixed point (in the $\sin \phi_{n^{*}}$ space), as is illustrated in Fig. 6 for some values of $K$.

We found numerically that for a given system the size of the basins of attraction is not evenly distributed among all fixed points: the stable solutions with phase locking $\sin \phi_{n^{*}}$ going to zero as $K \rightarrow \infty$ attract the majority of the initial conditions. Fortunately the behavior of the system for large values of $K$ is easier to analyze: in the limit $K \rightarrow \infty$ we have $\phi_{n}=\phi$, for $n=1, \ldots, N-1$, with $\phi$ given by the solutions of

$$
\sin [(N-1) \phi]=-\sin \phi,
$$

i.e.,

$$
\begin{gathered}
\phi_{\mathrm{I}}=\frac{2 \pi n_{1}}{N}, \quad n_{1}=0, \pm 1, \pm 2, \ldots, \\
\phi_{\mathrm{II}}=\frac{\left(2 n_{2}+1\right) \pi}{N-2}, n_{2}=0, \pm 1, \pm 2, \ldots
\end{gathered}
$$




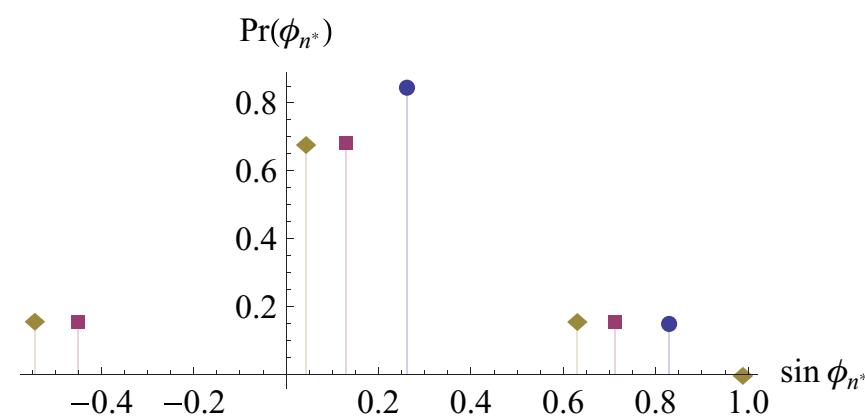

FIG. 6. (Color online) Estimation of the basin size for each of the stable fixed points within the $N=10$ case. Blue dots represent $K=$ 20, lilac squares represent $K=50$, and beige diamonds represent $K=120$

In this regime the solutions depend only on the system size $N$ and the values assumed by the discrete probability of reaching each of the stable fixed points (Fig. 7) are over the envelope function defined by $f\left(\sin \phi_{n^{*}}\right) \sim \exp \left(-a \sin ^{2} \phi_{n^{*}}\right)$.

\section{CONNECTION TO THE CHAIN}

In the previous sections we described the synchronized region of a ring of oscillators and showed how some of the properties of the chain are still present in this topology. Now we focus our attention to understand the appearance of multiple solutions. To do that we start from the solutions of a chain.

To construct the chain we remove the link between oscillators 1 and $N$ in the ring to end up with a chain of oscillators where the critical synchronization is defined by $\sin \phi_{n^{*}}=s \frac{K_{s}^{\text {chain }}}{K}$ (note that the $\sin \phi_{N}$ term does not enter in the dynamics). A saddle node bifurcation appears at $K=K_{s}^{\text {chain }}$ and naturally we have $\sin \phi_{n^{*}}=s$. The effect of closing the chain into a ring by connecting these two oscillators inserts the extra term present on the left-hand side of Eq. (6), which is a nonlinear function of $\phi_{n^{*}}$. Instead of a single solution for the fully synchronized state that extends for all $K \geqslant K_{s}$, this new configuration generates multiple stable solutions born at the local minima of the implicit multivalued function $K\left(\phi_{n^{*}}\right)$ that bifurcate into pairs of solutions as $K$ increases.

Now we build the ring from the open chain in a controlled way by coupling a continuous parameter $\alpha \in[0,1]$ to the interaction term $\sin \phi_{N}$ in Eq. (2). It is easy to see that in

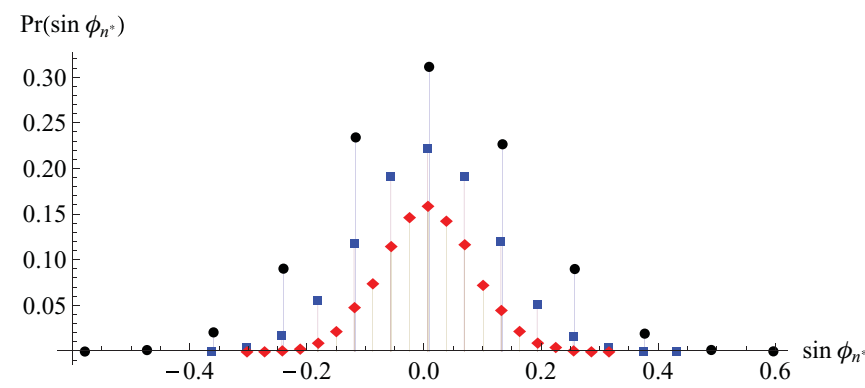

FIG. 7. (Color online) Discrete probability for the system to reach each of the stable fixed points (for large values of $K$ ) representing the estimation of the relative basin sizes. Black dots represent a system with $N=50$, blue squares represent $N=100$, and red diamonds represent $N=200$.
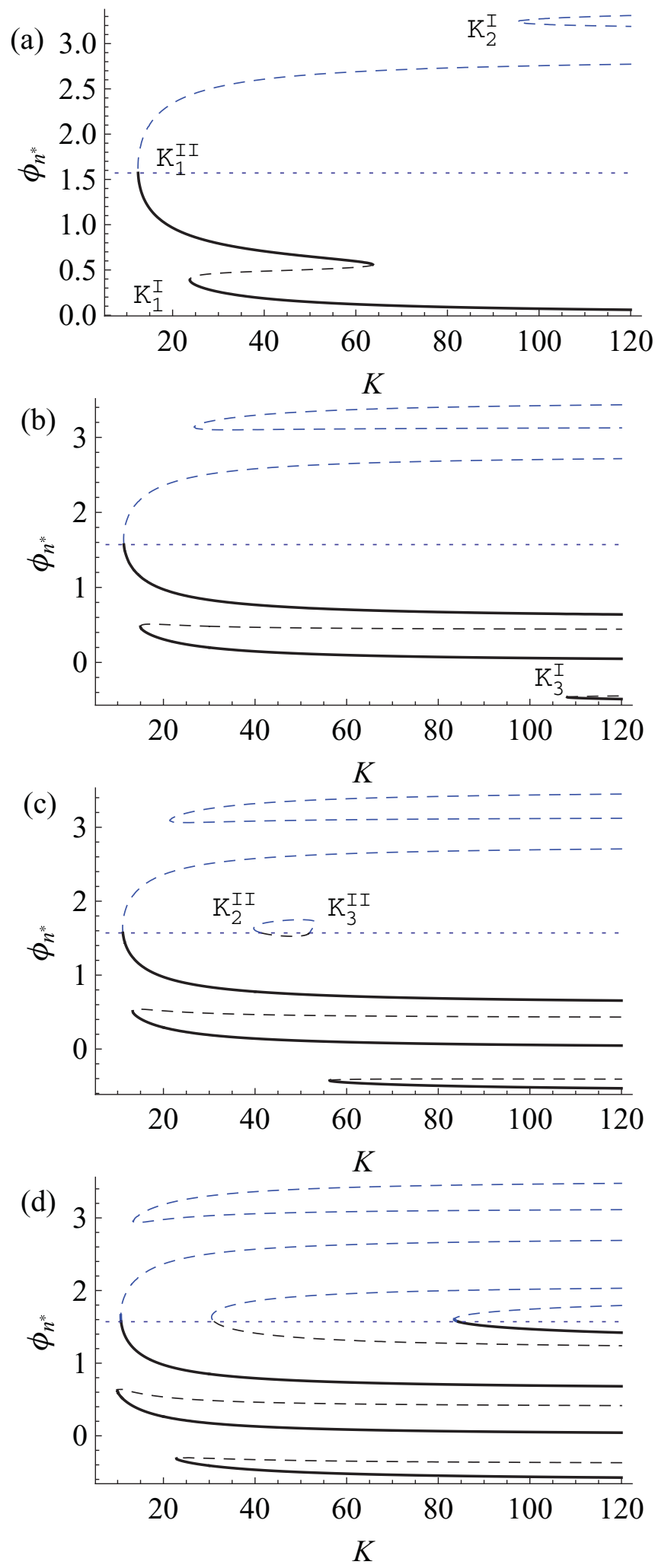

FIG. 8. (Color online) Bifurcation diagrams $\phi_{n^{*}}$ vs $K$ for $N=10$ as a function of the enclosing parameter $\alpha$. (a) Hysteresis formed after the stable solution loses stability as a function of $\alpha ; K_{2}^{\mathrm{I}}$ coming from infinity ( $\alpha=0.3$ ). (b) Turning point goes to infinity (bottom) creating two distinct solutions and $K_{3}^{\mathrm{I}}$ comes from infinity $(\alpha=0.5)$. (c) Birth of $K_{2}^{\mathrm{II}}$ and $K_{3}^{\mathrm{II}}$ from the closed circuit $(\alpha=0.7)$. (d) Bifurcation diagram of the ring topology showing all solutions on the region $(\alpha=1.0)$. The dotted lines correspond to $\phi_{n^{*}}=\pi / 2$. 


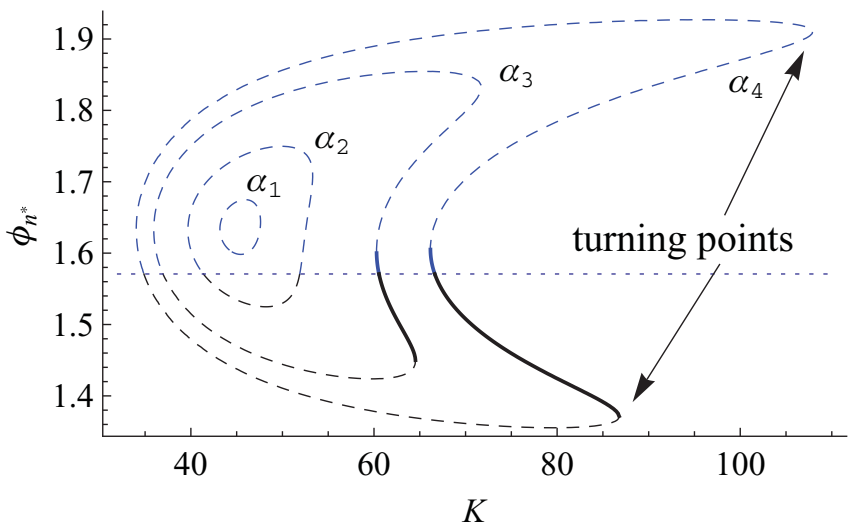

FIG. 9. (Color online) Closer look at the region of the birth of $K_{2}^{\mathrm{II}}$ and $K_{3}^{\mathrm{II}}$ showing solutions for four different values of $\alpha: \alpha_{1}=0.683$, $\alpha_{2}=0.7, \alpha_{3}=0.75$, and $\alpha_{4}=0.8$. The dotted lines correspond to $\phi_{n^{*}}=\pi / 2$.

the case of a chain $(\alpha=0) \sin \phi_{n^{*}}=s \frac{K_{s}^{\text {chain }}}{K}$ for all $K \geqslant K_{s}^{\text {chain }}$ [15]. The effect of increasing $\alpha$ deforms the original solution (as shown in Fig. 8) which in turn will generate the others observed for the ring. For small values of $\alpha$ a hysteresis appears at a fold bifurcation [Fig. 8(a)], creating the $K_{1}^{(\mathrm{I})}$ bifurcation from the stable branch born at $K_{1}^{(\mathrm{II})}$. As $\alpha$ increases the turning point goes to infinity [Fig. 8(b)], completely separating the original into two stable solutions. Bifurcations $K_{2}^{(\mathrm{I})}$ and $K_{3}^{(\mathrm{I})}$ do not present such turning points, as they seem to come from $K$ infinity [Figs. 8(a) and 8(b)].

Higher values of $\alpha$ give birth to a small closed circuit near $\phi_{n^{*}}=\pi / 2$ [Figs. 8(c) and 9], which via a deformation process

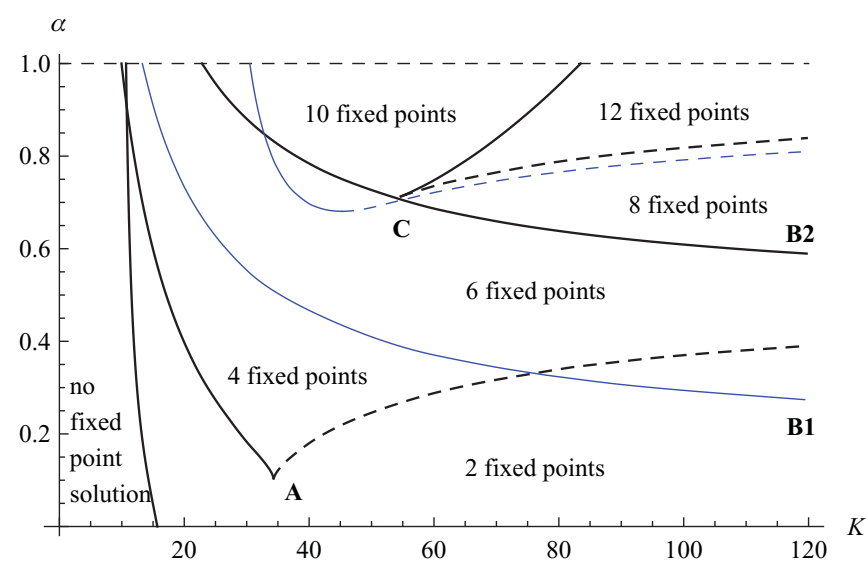

FIG. 10. (Color online) Stability diagram for the system. The birth of a pair of solutions is represented by a continuous line as one goes from lower to higher values of $K$, while the destruction of a pair of solutions is represented by dashed lines (the definitions are inverted if one goes from right to left). Thick (black) lines denote the creation of one stable solution and the thin (blue) ones account for the bifurcations with no stable equilibria. A: birth of the hysteresis. B1 and B2: bifurcations that come from infinity. C: Birth of the closed circuit that generates $K_{2}^{\text {(II) }}$ and $K_{3}^{\text {(II) }}$. increases the circuit until the turning points go to $K \rightarrow \infty$, creating $K_{2}^{(\mathrm{II})}$ and $K_{3}^{(\mathrm{II})}$, each one presenting two solutions. Figure 9 shows how the closed circuit of unstable solutions is born $\left(\alpha=\alpha_{1}\right)$ and as the parameter increases, the solution crosses the $\phi_{n^{*}}=\pi / 2$ line, giving rise to one stable solution. At this point the bubble starts to deform, creating the turning points on both sides of $\pi / 2$ and generating four solutions. When $\alpha=1$ the turning points go to infinity and the system presents all the properties of the ring previously described.

The complete structure of the synchronized state as the system is driven from a chain into a ring may be visualized on the stability diagram shown in Fig. 10: small values of $\alpha$ change the phase locking solutions $\phi_{n}$ but do not alter the main structure of the chain; for $\alpha \simeq 0.1$ and $K \simeq 35$ (region A) the properties of the ring start to become apparent as the hysteresis appears and the system presents bistability on a closed interval of values of $K$; regions $\mathbf{B} 1$ and $\mathbf{B 2}$ show the bifurcations $K_{2}^{(\mathrm{I})}$ and $K_{3}^{(\mathrm{I})}$ coming from infinity and the disappearance of the hysteresis; region $\mathbf{C}$ shows the birth of the closed circuit that creates $K_{2}^{(\mathrm{II})}$ and $K_{3}^{(\mathrm{II})}$.

\section{CONCLUSIONS}

In conclusion we studied a locally coupled Kuramoto model above the full synchronization transition for a ring of oscillators. We found a very rich panorama of solutions, although there is only one at the critical value for full synchronization. We were able to determine (analytically) the solvability region (SR) where the solutions exist and to show (numerically) that they all come from minima of the function $K\left(\phi_{n^{*}}\right)$, with $\phi_{n^{*}}$ being a specific chosen phase difference. From the observation that every bifurcation has a solution branch with a point tangent to the SR curves we were able to show that the multiplicity of solutions comes as distinct discrete values assumed by $\phi_{n^{*}}$ and $\phi_{N}$. The stability analysis of the solutions showed the existence of saddle node bifurcations responsible for the creation of both stable and unstable solutions. A statistical approach to estimating the size of the basin of attraction of the stable solutions was performed and we were able to observe that phase locking values of $\sin \phi_{n^{*}}$ closer to 0 (for large values of $K$ ) present the largest basins. Finally we studied a system with the coupling strength of a given link $\alpha$ varies from zero (free chain) to one (ring) to investigate the birth of these solutions. We observed two basic processes responsible for the generation of ring solutions from the open chain: deformations that creates hysteresis for a finite range of $\alpha$; spontaneous creation that either creates solutions coming from infinity or generates closed circuits with four solutions (only one being stable).

\section{ACKNOWLEDGMENTS}

F.F.F. thanks the IFT/UNESP for its hospitality. P.F.C.T. acknowledges support by CAPES (Brazil). The authors thank Dr. H.F. El-Nashar for fruitful discussions.
[1] H. Sakaguchi, S. Shinomoto, and Y. Kuramoto, Prog. Theor. Phys. 77, 1005 (1987).
[2] G. B. Ermentrout and N. Kopell, SIAM J. Math. Anal. 15, 215 (1984). 
[3] H. Daido, Phys. Rev. Lett. 61, 231 (1988).

[4] A. T. Winfree, Geometry of Biological Time (Springer, New York, 1990).

[5] C. W. Wu, Synchronization in Coupled Chaotic Circuits and Systems (World Scientific, Singapore, 2002).

[6] S. H. Strogatz, Sync: The Emerging Science of Spontaneous Order (Hyperion, New York, 2003).

[7] S. Manrubbia, A. Mikhailov, and D. Zanette, Emergence of Dynamical Order: Synchronization Phenomena in Complex Systems (World Scientific, Singapore, 2004).

[8] A. Arenas, A. Diaz-Guilera, J. Kurths, Y. Moreno, and C. Zhou, Phys. Rep. 469, 93 (2008).

[9] Y. Kuramoto, Chemical Oscillations, Waves and Turbulences (Springer, Berlin, 1984).

[10] A. S. Pikovsky, G. Osipov, M. G. Rosenblum, M. Zaks, and J. Kurths, Phys. Rev. Lett. 79, 47 (1997).

[11] J. A. Acebron, L. L. Bonilla, C. J. P. Vicente, F. Ritort, and R. Spigler, Rev. Mod. Phys. 77, 137 (2005).

[12] Zhigang Zheng, Gang Hu, and Bambi Hu, Phys. Rev. Lett. 81, 5318 (1998).

[13] Y. Zhang, G. Hu, and H. A. Cerdeira, Phys. Rev. E 64, 037203 (2001).

[14] Z. Liu, Y.-C. Lai, and F. C. Hoppensteadt, Phys. Rev. E 63, 055201(R) (2001).

[15] S. H. Strogatz and R. E. Mirollo, Physica D 31, 143 (1988).
[16] H. F. El-Nashar and H. A. Cerdeira, Chaos 19, 033127 (2009).

[17] P. Muruganandam, F. F. Ferreira, H. El-Nashar, and H. A. Cerdeira, Pramana 70, 1143 (2008).

[18] H. F. El-Nashar, P. Muruganandam, F. F. Ferreira, and H. A. Cerdeira, Chaos 19, 013103 (2009).

[19] B. C. Daniels, S. T. M. Dissanayake, and B. R. Trees, Phys. Rev. E 67, 026216 (2003).

[20] A. N. Pisarchik, R. Jaimes-Reátegui, J. R. Villalobos-Salazar, J. H. García-López, and S. Boccaletti, Phys. Rev. Lett. 96, 244102 (2006).

[21] J. Maurer and A. Libchaber, J. Phys. Lett. 41, L515 (1980).

[22] D. M. Heffernan, Phys. Lett. A 108, 413 (1985).

[23] J.-F. Vibert, A. S. Foutz, D. Caille, and A. Hugelin, Brain Res. 448, 403 (1988); A. Hunding and R. Engelhardt, J. Theor. Biol. 173, 401 (1995).

[24] P. Perlikowski, S. Yanchuk, M. Wolfrum, A. Stefanski, P. Mosiolek, and T. Kapitaniak, Chaos 20, 013111 (2010).

[25] U. Freudel, Int. J. Bifurcation Chaos Appl. Sci. Eng. 18, 1607 (2008).

[26] This assertion is difficult to prove analytically; however, we have performed detailed numerical calculations which show that they are saddle node bifurcations.

[27] The presence of symmetries on the $\{\omega\}_{N}$ configuration changes this fact, but these results will be published elsewhere. 